\title{
Mast Cell-Derived Exosomes Promote Th2 Cell Differentiation via OX40L-OX40 Ligation
}

\author{
Fei Li, Yuping Wang, Lihui Lin, Juan Wang, Hui Xiao, Jia Li, Xia Peng, \\ Huirong Dai, and Li Li
}

Department of Laboratory Medicine, Shanghai First People's Hospital, Shanghai Jiao Tong University School, Shanghai 200080, China

Correspondence should be addressed to Li Li; annylish@hotmail.com

Received 19 September 2015; Revised 27 January 2016; Accepted 11 February 2016

Academic Editor: Mario Clerici

Copyright ( $) 2016$ Fei Li et al. This is an open access article distributed under the Creative Commons Attribution License, which permits unrestricted use, distribution, and reproduction in any medium, provided the original work is properly cited.

Exosomes are nanovesicles released by different cell types, such as dendritic cells (DCs), mast cells (MCs), and tumor cells. Exosomes of different origin play a role in antigen presentation and modulation of immune response to infectious disease. In this study, we demonstrate that mast cells and $\mathrm{CD}^{+}{ }^{+} \mathrm{T}$ cells colocated in peritoneal lymph nodes from BALB/c mouse. Further, bone marrow-derived mast cells (BMMCs) constitutively release exosomes, which express CD63 and OX40L. BMMC-exosomes partially promoted the proliferation of $\mathrm{CD} 4^{+} \mathrm{T}$ cells. BMMC-exosomes significantly enhanced the differentiation of naive $\mathrm{CD} 4^{+} \mathrm{T}$ cells to Th2 cells in a surface contact method, and this ability was partly inhibited by the addition of anti-OX40L Ab. In conclusion, BMMC-exosomes promoted the proliferation and differentiation of Th2 cells via ligation of OX40L and OX40 between exosomes and $\mathrm{T}$ cells. This method represents a novel mechanism, in addition to direct cell surface contacts, soluble mediators, and synapses, to regulate $\mathrm{T}$ cell actions by BMMC-exosomes.

\section{Introduction}

Exosomes are 30 to $100 \mathrm{~nm}$ extracellular membrane vesicles of endocytic origin, which are released into the extracellular environment upon fusion of multivesicular bodies with the plasma membrane. They were first reported in vitro in sheep reticulocytes by Johnstone et al. [1]. Subsequent reports showed that a range of cells including DCs, B cells, T cells, and tumor cells secreted exosomes in vitro and in vivo. Further, exosomes also occur naturally in body fluids such as blood, urine, saliva, and breast milk [2-6].

Although they share similar morphology, exosomes are unique in their protein composition. Because of their endosomal origin, all exosomes contain membrane transport and fusion proteins (GTPases, annexins, and flotillin), tetraspanins (CD9, CD63, CD81, and CD82), heat shock proteins (Hsp70 and Hsp90), proteins involved in multivesicular biogenesis (Alix and TSG), and lipid-related proteins and phospholipases $[7,8]$. In addition to these membrane proteins, over 4400 different proteins have been identified in association with exosomes $[9,10]$. Among these proteins, exosomes of different cellular origin or similar cells but in different states harbor different protein sets [11, 12]. For example, mast cell-derived exosomes carry c-kit and $\operatorname{IgE}$ receptors [13], and B cell-derived exosomes contain MHC II on the membrane [10]. Additionally, more MHC II, CD80, CD86, and other costimulatory molecules were detected in mature DCs-derived exosomes than in immature DCs [12]. A few studies suggested that the protein repertoire of exosomes was different from that of their parental cells [14]. Exosomes have been reported to contain significant amounts of RNAs, including miRNAs and mRNAs, but not DNA, and contain RNAs not detected in their parental cells [15].

Due to the existence of proteins and nucleic acid components, exosomes are supposed to play an important physiological and pathological role. In the last decade, much emphasis was placed on the effect of exosomes on immunological regulation and tumor development. Exosomes from mature DCs promoted the action of T cells, while those from immature DCs mediated immune tolerance [16]. Exosomes derived from bronchoalveolar lavage fluid suppressed allergic asthma in mouse models [17]. Tumor-derived exosomes promoted 
the proliferation of $\mathrm{CD} 8^{+} \mathrm{T}$ cells, which eliminated tumor cells [18]. These data indicate that exosomes contribute to the maintenance of health and are involved in the development of disease.

Mast cells are known as effector cells in the development of IgE-mediated allergic asthma. Degranulation after activation leads to secretion of bioactive substances, such as histamine, prostaglandin, and proteases to induce allergic response. Further, they also play a role in innate and adaptive immunity via secretion of cytokines such as TNF- $\alpha$, IL4, and IL-13. Mast cell-derived exosomes have been found to participate in immune regulatory response. To date, the effects of mast cell-derived exosomes on DCs, T cells, and B cells have been reported extensively. For example, mast cellderived exosomes altered the phenotype of DCs in vitro [19]. In this study, we sought to determine the effects of exosomes from bone marrow-derived mast cells on naive $T$ cells and the possible mechanisms.

\section{Materials and Methods}

2.1. Mice. BALB/c mice (5-wk-old) were purchased from Sion-British Sippr/BK Laboratory and housed in the Animal Experimental Center of Shanghai First People's Hospital (Shanghai, China) under specific pathogen-free conditions. The Chancellor's Animal Research Committee approved all the animal studies and confirmed that the experiments involving animals adhered to the guidelines set forth by the Shanghai Jiao Tong University School of Medicine (Shanghai, China).

2.2. Reagents and Antibodies. Fetal bovine serum (FBS), RPMI1640, and fluorescence dyes Dio and Dil were purchased from Life Technologies (California, USA). Recombinant mIL-3 and mIL-4 were purchased from PeproTech (Rocky Hill, NJ, USA). CD4 ${ }^{+} \mathrm{CD}^{2} \mathrm{~L}^{+} \mathrm{T}$ cell Isolation Kit II was purchased from Miltenyi Biotec (Paris, France). FITClabeled rat anti-mouse mAbs directed against CD117, PElabeled rat anti-mouse $\mathrm{mAbs}$ directed against FceRI, FITClabeled rat anti-mouse mAbs directed against CD4, PElabeled rat anti-mouse mAbs directed against IL-4, and PerCP/C $\gamma 5$.5-labeled rat anti-mouse $\mathrm{mAbs}$ directed against IFN- $\gamma$ were purchased from Biolegend (San Diego, CA). Goat anti-mouse OX40 mAb and rat anti-mouse OX40L $\mathrm{mAb}$ were obtained from R\&D System (Minneapolis, MN, USA). Cell Counting Kit -8 (CCK-8, DojinDo, Japan) was used to assess the proliferation rate of cells. Antimast cell tryptase antibody was purchased from Abcam (America). Anti-rat IgG-HRP was purchased from Dako (Japan). ECL+ system was purchased from Amersham (Piscataway, NJ). All the information of primary antibodies is included in Table 1.

2.3. Preparation of Cells. BMMCs were prepared as previously described. After 4 wk of culture using RPMI 1640 supplemented with $10 \%$ heat-inactivated FBS and $10 \mathrm{ng} / \mathrm{mL}$ rIL-3, cells were harvested and consisted of $98 \%$ pure MCs as assessed by toluidine blue staining, CD117 and IgE receptor
TABLE 1: Antibody profile.

\begin{tabular}{lcccc}
\hline Antibody & Company & ID number & Clone & Applications \\
\hline Tryptase & Abcam & ab2378 & AA1 & IHC, IF \\
CD4 & Biolegend & 100401 & GK1.5 & IHC, FC, IP \\
CD4 & Biolegend & 100406 & GK1.5 & FC \\
CD117 & Biolegend & 105806 & $2 B 8$ & FC \\
FcERI & Biolegend & 134301 & MAR1 & FC \\
IL-4 & Biolegend & 144804 & $1015 F 8$ & FC \\
IFN- $\gamma$ & Biolegend & 127302 & MAR1-5A3 & FC \\
OX40 & R\&D & AF1256-SP & P47741 & WB \\
OX40L & R\&D & MAB1236-SP & 182601 & WB \\
$\beta$-actin & Proteintech & HRP-60008 & ag0297 & ELISA, WB
\end{tabular}

WB: Western blot; IHC: immunohistochemistry; FC: flow cytometry; IF: immunofluorescence; ELISA: enzyme-linked immunosorbent assay; IP: immunoprecipitation.

(FceRI) expression. BMMCs were cultured for the last $72 \mathrm{~h}$ before harvest in the presence of RPMI1640 complemented with $10 \mathrm{ng} / \mathrm{mL}$ rIL-3 and FBS depleted of exosomes, allowing the use of supernatant containing only MC-derived exosomes.

Naive $\mathrm{CD}^{+}{ }^{+} \mathrm{T}$ cells were negatively selected from the spleen cells by $\mathrm{CD} 4^{+} \mathrm{CD} 62 \mathrm{~L}^{+} \mathrm{T}$ cell Isolation Kit II. According to the manufacturer's instructions, the non-T-helper cells as well as regulatory T cells and $\gamma / \delta \mathrm{T}$ cells were depleted by indirect magnetic labeling using a cocktail of lineage-specific biotin-conjugated antibodies against CD8a, CD45R, CD49b, CD11b, and Ter-119, as well as antibodies against CD25 and TCR $\gamma / \delta$ in combination with Anti-Biotin MicroBeads. Subsequently, $\mathrm{CD} 4^{+} \mathrm{CD} 62^{+} \mathrm{T}$ cells were positively selected from the enriched $\mathrm{CD} 4^{+}$helper cell fraction with $\mathrm{CD} 62 \mathrm{~L}$ MicroBeads.

2.4. Exosomes Isolation. Exosomes were prepared from the supernatant of 4-wk-old BMMCs cultures [15]. During the last $72 \mathrm{~h}, \mathrm{BMMCs}$ were cultured at $3 \times 10^{6}$ cells $/ \mathrm{mL}$ inIL-3containing RPMI 1640. Supernatants were then subjected to two successive centrifugations at $300 \mathrm{~g}$ for $5 \mathrm{~min}$ and at $1,200 \mathrm{~g}$ for $20 \mathrm{~min}$ to eliminate cells and debris. Exosomes were purified by filtration of $0.22 \mu \mathrm{g}$ pore filters, followed by a centrifugation for $2 \mathrm{~h}$ at $120,000 \mathrm{~g}$. Two fractions were obtained: a high-density (pellet) and a low-density (hypodense) fraction. The exosomes concentrated in the pellet were washed twice in a large volume of PBS centrifuged at $120,000 \mathrm{~g}$ for $2 \mathrm{~h}$. The preparation of exosomes was stored under $-80^{\circ} \mathrm{C}$.

2.5. Coculture of Exosomes and Naive T Cells. Before coculture, 24-well plates were coated with anti-mouse CD3 and $\mathrm{CD} 28 \mathrm{mAb}$ both at $10 \mu \mathrm{g} / \mathrm{mL}$ overnight at $4^{\circ} \mathrm{C} .1 \times 10^{6}$ naive $\mathrm{T}$ cells were incubated in 24 -well plates with exosomes derived from $1 \times 10^{6}$ or $1 \times 10^{7}$ mast cells in $1 \mathrm{~mL}$ RPMI1640 supplemented with $10 \%$ exosomes-free FBS and $10 \mathrm{ng}$ rlL4 for $72 \mathrm{~h}$. Alternatively, some groups were added into complete medium as blank control or $10 \mu \mathrm{g} / \mathrm{mL}$ anti-mouse OX40L mAb according to the experiment. The assessment 
of proliferation of $\mathrm{T}$ cells was conducted by CCK-8 Kit according to manufacturer's protocol. In the last 4 hours, $100 \mathrm{ng}$ PMA and $1 \mu \mathrm{g}$ A23187 were added into the medium, and, 2 hours later, $1 \mu \mathrm{L}$ GolgiStop was added to suppress the release of cytokines from plasma. In some experiments, naive $T$ cells stained with Dio were cultured with exosomes stained with Dil.

2.6. Flow Cytometric Analysis. BMMCs were washed twice with PBS and stained with FITC-labeled CD117 and PElabeled IgE receptor for $20 \mathrm{~min}$ under room temperature. Washed again, the samples were subjected to flow cytometry (Cytomics FC500, Beckman Coulter). For analysis of differentiation of T cells, cells were collected, washed with PBS twice, and stained with FITC-labeled anti-mouse CD4 mAb for $20 \mathrm{~min}$ under room temperature. Followed by fixation and permeabilization, intracellular cytokines IL- 4 and IFN- $\gamma$ were stained. Then FACS was performed to identify Thl and Th2 cells.

2.7. Western Blotting. Exosomes were incubated for $30 \mathrm{~min}$ on ice in lysis buffer (PBS containing RIPA and protease inhibitors). In addition, cell lysates (1 million cells per $100 \mu \mathrm{L}$ of lysis buffer) from mast cells were also prepared. Insoluble material was removed by centrifugation $(10,000 \mathrm{~g}$ for $10 \mathrm{~min}$ ), and the protein concentration of supernatants was determined by protein assay. Protein concentrations of ultracentrifuged exosomes were again determined by $\mathrm{BCA}$ assay. Equal quantity of exosomes or cellular protein $(5 \mu \mathrm{g})$ was loaded per well of $10 \%$ acrylamide gels, and after electrophoresis proteins were transferred to PVDF membranes and blocked for 2 hours in PBS containing 3\% BSA-TBST and $0.05 \%$ Tween-20. Primary antibodies were incubated for 2 hours and washed four times, followed by 1 hour of incubation with anti-rat IgG-HRP used at1 in 6,000 dilution in BSA-TBST. Detection of bands was performed with the ECL+ system.

2.8. Immunohistochemistry. Peritoneal lymph nodes were collected from healthy mouse and five-micrometer paraffinembedded sections were prepared. Slides were stained with $\mathrm{CD}^{+}$, tryptase, and DAPI recorded by fluorescence microscope (Zeiss). In addition, slides were, respectively, stained with $\mathrm{CD} 4$ and tryptase to assess $\mathrm{T}$ cells and mast cells.

2.9. Electron Microscopy. Exosomes were examined using negative staining as described in [20]. Briefly, the purified exosomes pellet was fixed in $2 \%$ paraformaldehyde at $4^{\circ} \mathrm{C}$, and $5 \mu \mathrm{L}$ deposited on Formvar coated EM grids. After 20 min of absorption, grids were rinsed in PBS and fixed with $1 \%$ glutaraldehyde for $5 \mathrm{~min}$. After washing in distilled water, grids were contrasted with uranyl oxalate $(75 \mathrm{mM}$ oxalic acid in $2 \%(\mathrm{w} / \mathrm{v})$ uranyl acetate and final $\mathrm{pH}$ adjusted to 7.0 with $\left.\mathrm{NH}_{4} \mathrm{OH}\right)$ and embedded in $4 \%(\mathrm{w} / \mathrm{v})$ uranyl acetate and $2 \%(\mathrm{w} / \mathrm{v})$ methylcellulose for transmission electron microscopy (Hitachi).
2.10. Statistical Analysis. Results were expressed as mean $\pm \mathrm{SD}$. Comparisons between mean values were performed by ANOVA, followed by the Student-Newman-Keuls (SNK) test. A value of $p<0.05$ was considered significant.

\section{Results}

3.1. Colocalization of Mast Cells and CD $4^{+} \mathrm{T}$ Cells in Peritoneal Lymph Node. In previous studies, mast cells were associated with $\mathrm{T}$ cell activation in the immune response to resistant parasite infections as well as in allergic response [21, 22]. Further, these two cells were found to colocalize in intestinal tissues [23]. In the present study, we found that mast cells and $\mathrm{CD} 4^{+} \mathrm{T}$ cells coexisted in peritoneal lymph nodes of healthy mice and were closely linked (Figures $1(\mathrm{a})$ and $1(\mathrm{~b})$ ). When lymph node sections were stained with CD4 and tryptase, respectively, the shape of the $\mathrm{CD} 4^{+} \mathrm{T}$ cells was regular and clear, while mast cells were blurred, with brown particles observed outside the cells (Figures 1(c) and 1(d)). These data indicate that the mast cells potentially modulate the actions of $\mathrm{CD} 4^{+} \mathrm{T}$ cells.

3.2. Characteristics of Bone Marrow Mast Cells and Their Exosomes. Mast cell-derived exosomes regulate immune response. In order to explore the effects of mast cellexosomes on $\mathrm{CD}^{+} \mathrm{T}$ cells, mast cells from bone marrow were generated in the presence of IL-3 following a 4-week culture. Figure 2(a) illustrates the morphology of BMMCs containing an abundance of purple granules after toluidine blue staining. Flow cytometry analysis was also performed to identify mast cells based on the expression of CD117 and IgE receptor, suggesting that over $98 \%$ of cells were mast cells (Figure 2(b)). The BMMC-exosomes were isolated by successive centrifugation. Electron microscopy showed that BMMC-exosomes were 60-100 $\mathrm{nm}$ in diameter and displayed a cup-shape morphology (Figure 2(c)).

The Western blot indicated that exosomes expressed CD63, a marker of membrane structure (Figure 2(d)). The data were consistent with the description of exosomes based on size and morphology, suggesting that BMMCs constitutively released exosomes.

3.3. BMMC-Exosomes Promote Th2 Differentiation. To investigate the effects of BMMC-exosomes on the proliferation and differentiation of $\mathrm{T}$ cells, naive $\mathrm{CD}^{+} \mathrm{T}$ cells were coincubated with BMMC-exosomes in the presence of IL4 (see Section 2.5) for 72 hours in 24-well plate precoated with anti-CD3 and anti-CD28 mAb. Compared with the blank control without exosomes in culture medium, $\mathrm{T}$ cells in the exosome group showed a higher proliferation rate at different exosome/T ratio (Figure 3(a)). In the following experiments, we used $\mathrm{T}$ cells and mast cells providing exosomes, in the ratio of $1: 1$. Surprisingly, we found that approximate $28 \%$ of cells were Th2 cells, which were IL4 positive, while the blank control group constituted 13\% 


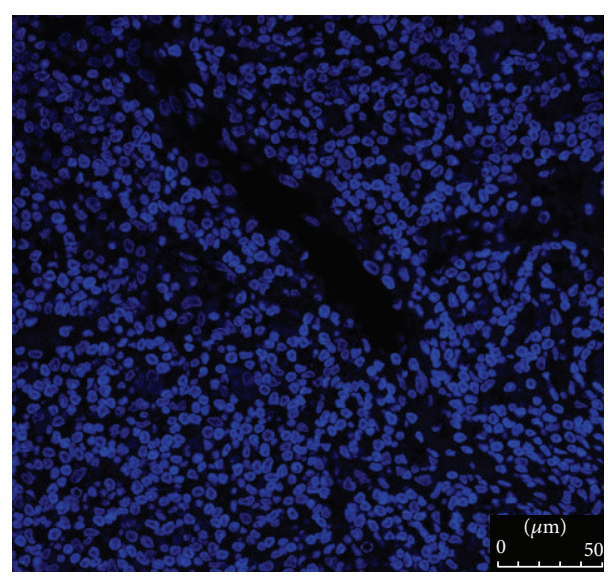

(a)

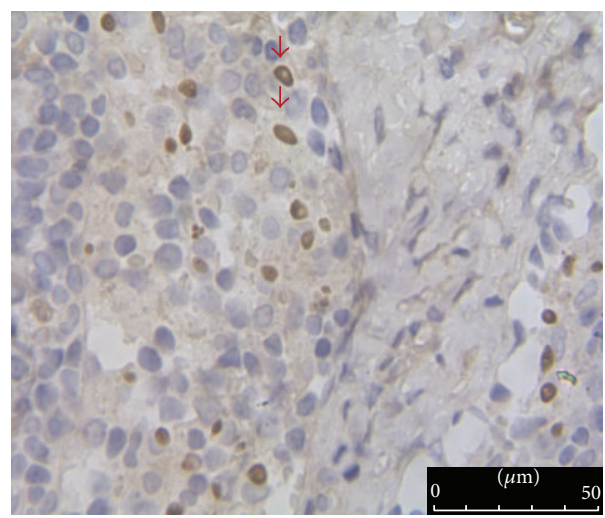

(c)

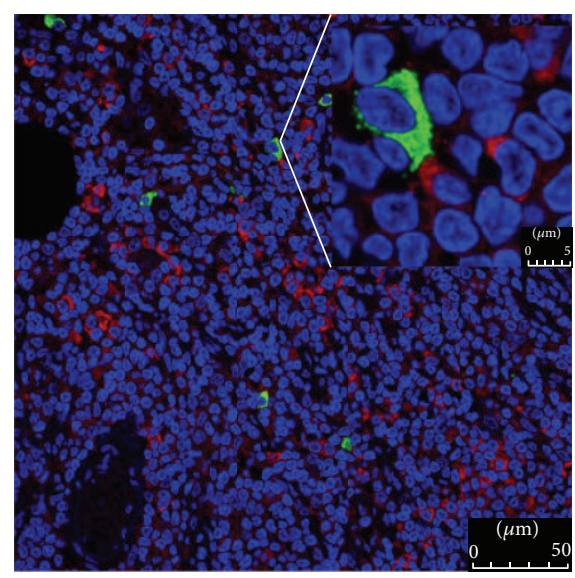

(b)

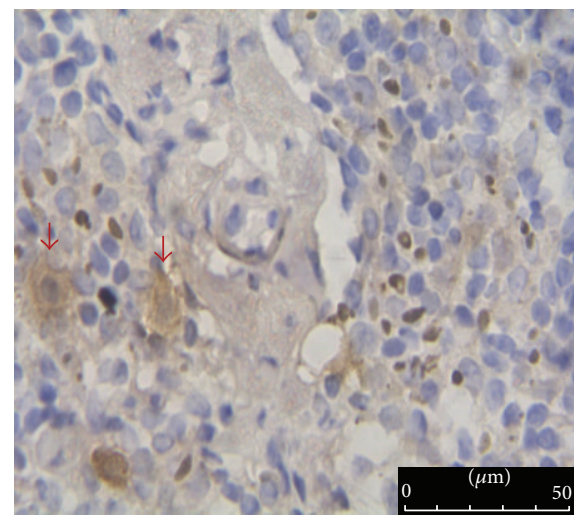

(d)

FIGURE 1: Location of mast cells and $\mathrm{CD}^{+}{ }^{+} \mathrm{T}$ cells as well as their morphology in peritoneal lymph node. (a) As a negative control, the section of lymph node was incubated with PBS instead of primary antibody (200x); (b) mast cells (green, stained with antimast cell tryptase antibody) and $\mathrm{CD}^{+} \mathrm{T}$ cells (red, stained with anti-CD4 antibody) colocalized in the peritoneal lymph node, marked by the red arrows (200x); (c) as a control, the outline of $\mathrm{CD}^{+} \mathrm{T}$ cells is clear $(400 \mathrm{x})$; (d) mast cells are blurred and surrounded by tiny brown particles $(400 \mathrm{x})$. Scale bars are $50 \mu \mathrm{m}$. In this experiment, CD4 and tryptase are used to mark $\mathrm{CD} 4^{+} \mathrm{T}$ cells and mast cells, respectively.

of Th2 cells in the whole $\mathrm{CD}^{+}$cells (Figures $3(\mathrm{~b})-3(\mathrm{e})$ ). These results demonstrated that BMMC-exosomes promoted significant proliferation of $\mathrm{T}$ cells. Importantly, BMMCexosomes enhanced the differentiation of naive $\mathrm{CD} 4^{+} \mathrm{T}$ cells to Th2 cells in vitro. These findings support the perspective that exosomes from mast cells modulated immune response.

3.4. BMMC-Exosomes Attached to $C D 4^{+} T$ Cells. Previous studies reported that exosomes affected other cells in different ways. Morelli et al. reported that DCs internalized exosomes via endocytosis [24]. Another group found that DC-derived exosomes played a role in T cells by ligation of LFA-1 and ICAM-1 [25]. Further, exosomes may break down and release contents into the environment, to exert their functions. To clarify the association and localization of BMMC-exosomes within $\mathrm{T}$ cells, we labeled the $\mathrm{T}$ cells and exosomes with different fluorescent dyes and measured the dynamics over the first 24 hours. As shown in Figure 4, BMMC-exosomes adhered to the surface of $\mathrm{T}$ cells at 12 hours after coculturing.
Even though the dish was swayed slightly, few exosomes separated from T cells. During the 24 hours, no exosomes were taken up by $\mathrm{T}$ cells. We, therefore, postulated that BMMC-exosomes may affect the action of T cells by direct surface contact or by release of their soluble contents, but not by endocytosis.

3.5. BMMC-Exosomes Function on $C D 4^{+} T$ Cells via Ligation of OX4OL and OX40. We investigated the molecular mechanism by which BMMC-exosomes promoted the differentiation of naive $\mathrm{CD} 4^{+} \mathrm{T}$ cells to Th 2 cells. Earlier studies demonstrated that IL-4, ICAM-1, CD40, and histamine facilitated differentiation of naive $\mathrm{CD} 4^{+} \mathrm{T}$ cells to Th2 cells. In addition, DCs enhanced the differentiation of Th2 cells via ligation of OX40L/OX40 between DCs and T cells [26]. MC activated $\mathrm{T}$ cells by OX40L [27]. Therefore, we wondered whether OX40L/OX40 played a role in the differentiation of $\mathrm{T}$ cells stimulated by BMMC-exosomes. The presence of OX40L was first determined in BMMC and BMMC-exosomes by Western blot, although it was lower in exosomes than in 


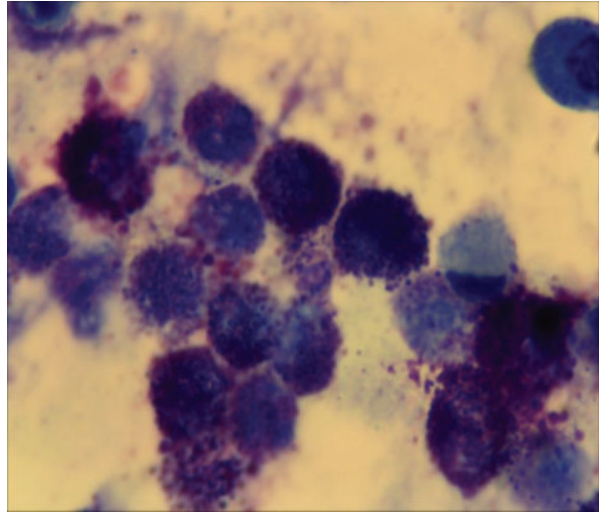

(a)

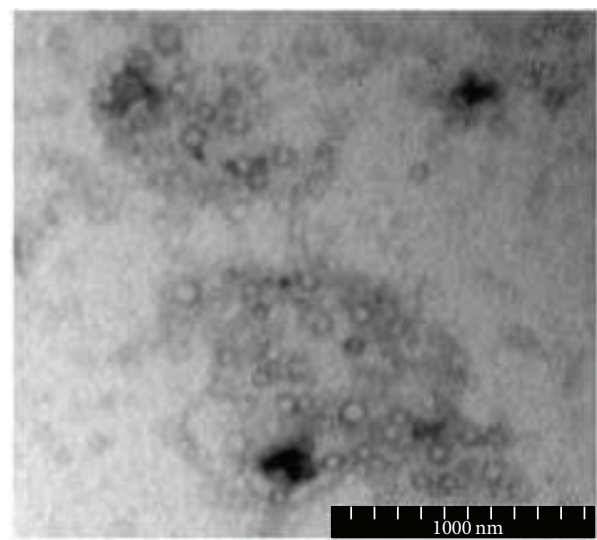

(c)

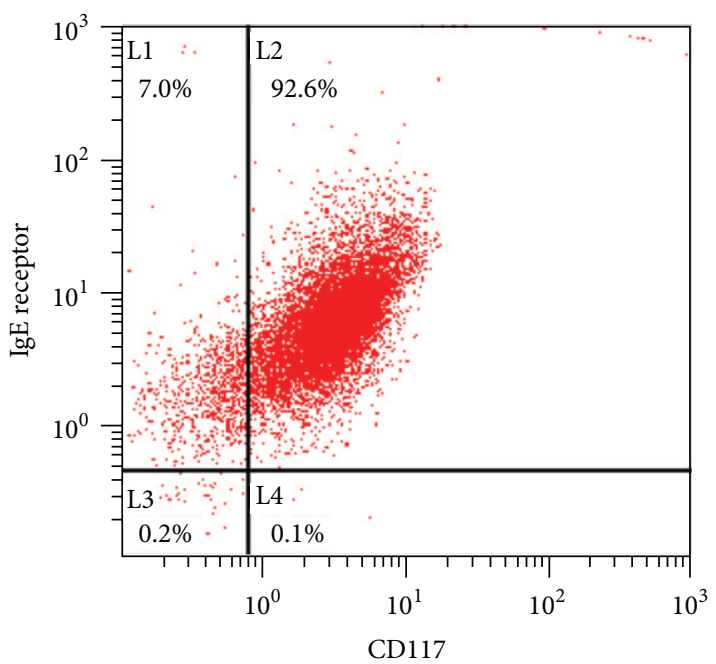

(b)

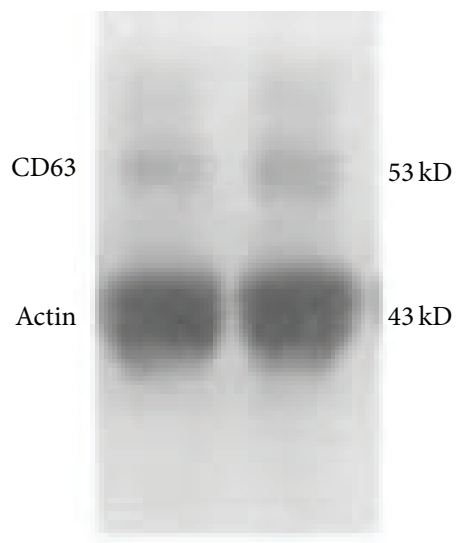

(d)

Figure 2: Characteristics of bone marrow mast cells and their exosomes. (a) Bone marrow mast cells showed abundant purple granules following toluidine blue staining (400x). (b) Mast cells were positive for CD117 and IgE receptor (FceRI) by flow cytometry analysis. (c) BMMC-exosomes displayed a cup-shape morphology; scale bar $=100 \mathrm{~nm}$. (d) Western blot indicated that BMMC-exosomes expressed CD63, a marker constitutively expressed by exosomes.

parental cells (Figure 5(a)). Further, OX40 was detected on the surface of T cells by FACS (Figure 5(b)). In the following experiments, anti-mouse OX40L mAb was added into the culture system to block OX40L on the exosomes. Compared with the exosome group, the group treated with exosomes and anti-OX40L mAb showed a lower number of Th2 cells. However, the inhibition of OX40L failed to completely inhibit the differentiation of Th2 cells to the level in the control group (Figures 5(c)-5(f)). These results demonstrated that OX40L on BMMC-exosomes ligated with OX40 on the surface of $\mathrm{T}$ cells to stimulate naive $\mathrm{CD} 4^{+} \mathrm{T}$ cell differentiate to Th 2 cells. Other factors also promoted the differentiation of Th2 cells.

\section{Discussion}

Mast cells are ubiquitously distributed among tissues, including bone marrow and lymphoid tissue. For several decades, human mast cells have been established as the key effector cells in allergic inflammation. They bind IgE on their surface by expressing the high-affinity $\mathrm{Fc}$ receptor for $\operatorname{IgE}$ and release histamine and other mediators after crosslinking of surface-bound IgE by allergen. Currently, mast cells are viewed similar to lymphocytes and other major immune cells involved in host defense and homeostasis. Previous studies in mice and rats and to some extent in humans have shown that mast cells play a central role in host defense against bacteria and parasites, via the release of cytokines and other mediators that recruit neutrophils, eosinophils, and Th2 cells to the site of infection [28]. Further, mast cells have been identified in Zebrafish, with a hematopoietic system, which is highly conserved with human beings [29]. To date, few studies have reported allergic responses in fish. Therefore, mast cells may play other important roles in homeostasis, in addition to allergic inflammation. In this study, we found mast cells and $\mathrm{CD}^{+} \mathrm{T}$ cells colocalized in the peritoneal 


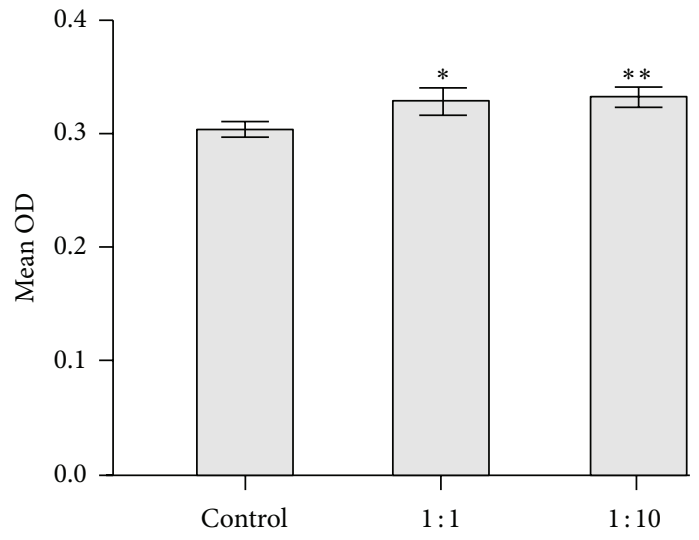

(a)

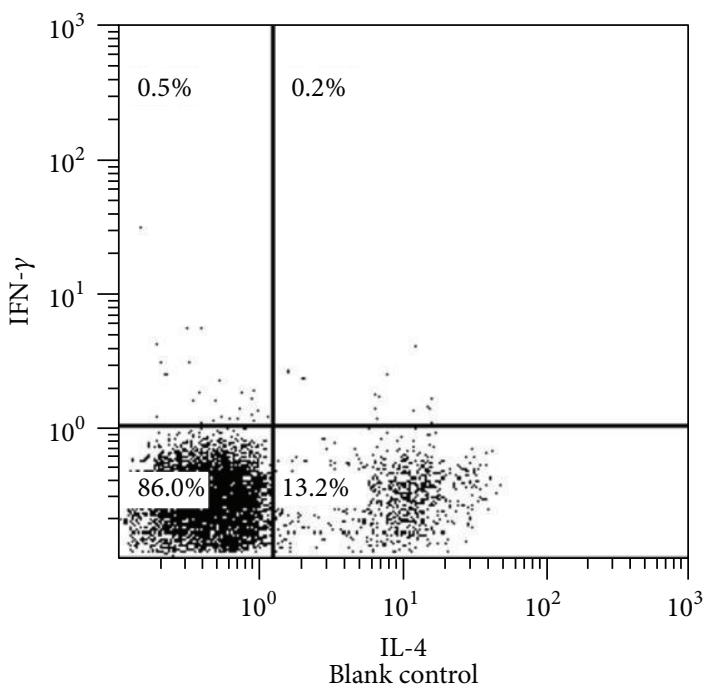

(c)

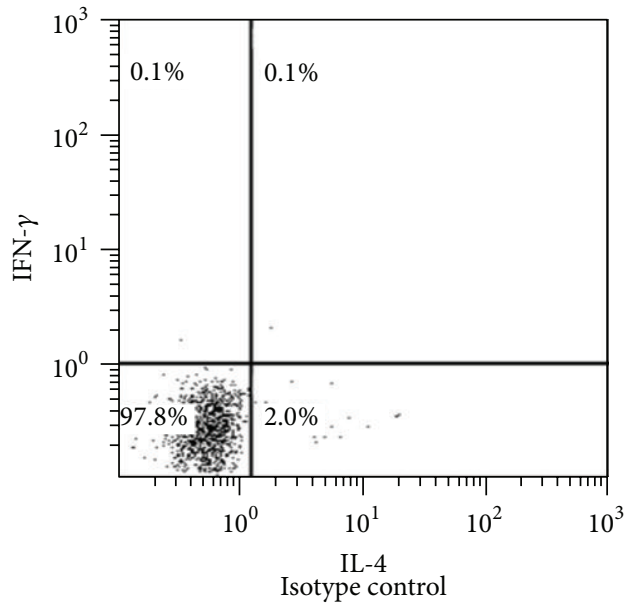

(b)

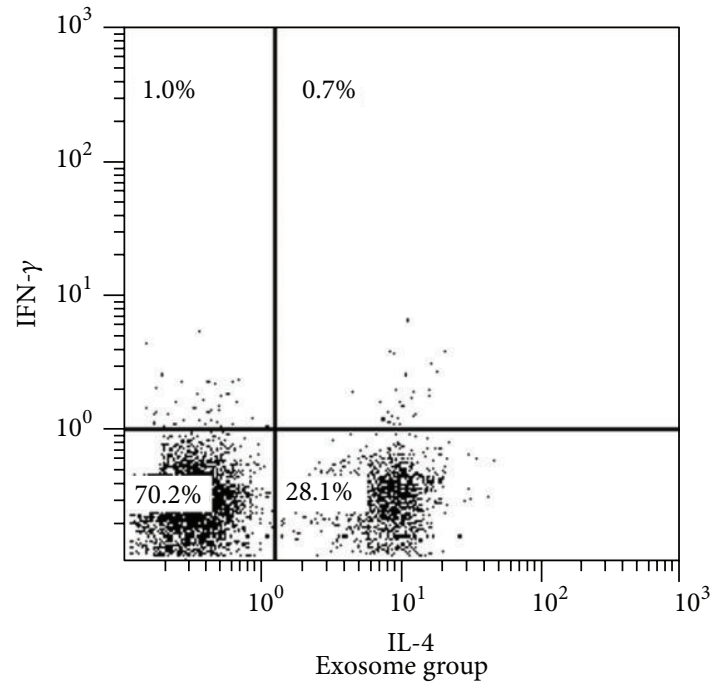

(d)

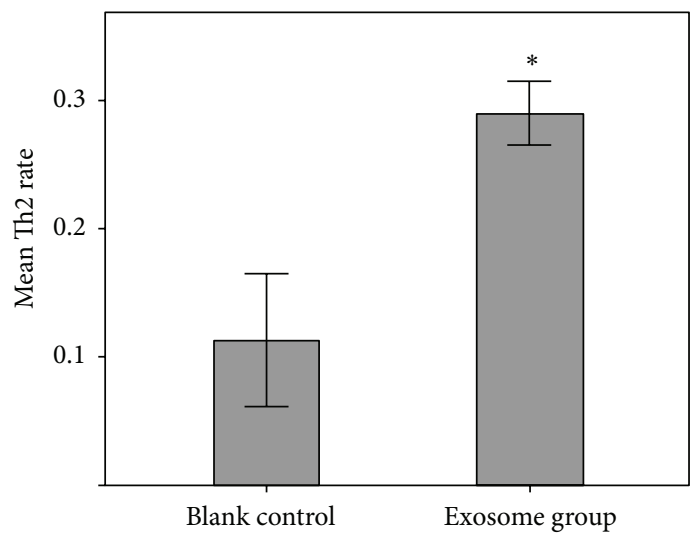

(e)

Figure 3: Effects of BMMC-exosomes on naive $\mathrm{CD} 4^{+} \mathrm{T}$ cells. (a) $10^{6}$ naive $\mathrm{CD} 4^{+} \mathrm{T}$ cells were coincubated with BMMC-exosomes generating $10^{6}$ or $10^{7}$ BMMCs under Th2 conditions. Compared with the blank control without exosomes, groups containing different levels of exosomes showed higher proliferation. T cell proliferation was quantified by incorporation of CCK-8. Results are expressed as mean O.D. at $450 \mathrm{~nm}$ and are representative of three experiments. $*$, compared with control, $p<0.05$. **, compared with $1: 1$ group, $p>0.05$. Student-Newman-Keuls (SNK) test was used. (b-d) After $72 \mathrm{~h}$ of culturing, the exosome group showed over $28 \%$ of Th2 cells, which was about twice the control group. (e) The rate of Th2 cells in the T cells was expressed as mean $\pm \mathrm{SD} *$, compared with blank control, $p<0.05$. The results represented four independent experiments. 


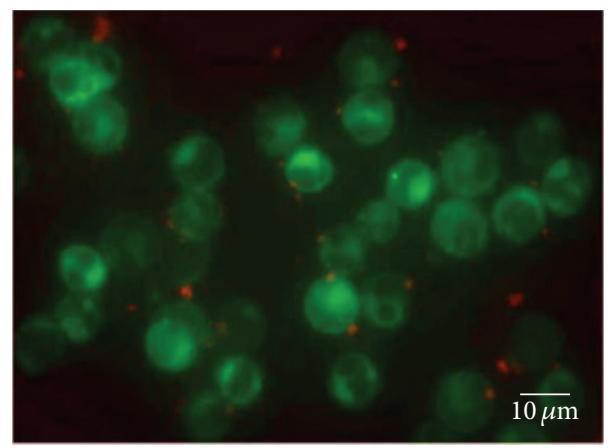

FIgure 4: Mechanism of BMMC-exosome function in naive CD4 ${ }^{+} \mathrm{T}$ cells. Exosomes and $\mathrm{T}$ cells were stained with Dil (red-orange) and Dio (green), respectively. During the first 24 hours, exosomes were found to adhere to T cells and no endocytosis was observed. The picture was taken 12 hours after the coculture at a magnification $\times 400$.

lymph node and attached to each other in some positions. Earlier reports also indicated that mast cells were found close to $\mathrm{T}$ cells at the intestinal tissue of patients with inflammatory bowel disease [23]. In addition, Wang et al. reported that mast cells migrated to lymph nodes in infections [30]. These findings suggested that mast cells reacted with $\mathrm{T}$ cells.

Referring to the interaction between mast cells and $\mathrm{T}$ cells, most investigators focused on mast cell function on $\mathrm{T}$ cells. Dimitriadou et al. found that mast cells captured antigens and presented them to T cells in MHC II-dependent manner, to activate $\mathrm{T}$ cells [31]. In another study, mast cells were reported to promote the differentiation of $\mathrm{T}$ cells to $\mathrm{Th} 2$ cells by release of histamine and TNF [32]. Since the discovery of exosomes, the effects of mast cell-derived exosomes on $\mathrm{T}$ cells have been explored in different conditions. When coincubated with spleen cells, mast cell-exosomes induced the activation of lymphocytes and production of IFN- $\gamma$, IL2 , and $\operatorname{IgG}[33]$. In order to explore the effects of BMMCexosomes on naive $\mathrm{CD}_{4}^{+} \mathrm{T}$ cells, we isolated naive $\mathrm{CD} 4^{+}$ T cells using MACS column system and coincubated them with BMMC-exosomes. Interestingly, we found that BMMCexosomes promoted differentiation of naive $\mathrm{CD} 4^{+} \mathrm{T}$ cells to Th2 cells significantly, when incubated with IL-4, which was not consistent with the results of Skokos et al. study [33]. The discrepancy may be attributed to the culture conditions. In our system, purified naive $\mathrm{CD} 4^{+} \mathrm{T}$ cells were incubated under Th2 condition, while Skokos et al.'s study incubated mixed spleen cells under neutral conditions without IL-4. In fact, we coincubated naive $\mathrm{CD} 4^{+} \mathrm{T}$ cells with BMMC-exosomes in the neutral condition, but no Th1 or Th2 cells were detected (data not shown). This result was consistent with the control group without exosomes and IL-4. Therefore, we speculated that the effect of BMMC-exosomes on T cells to a large extent depended on culture conditions.

For several decades, cell-cell communications were thought to occur by receptor-mediated events, either by recognizing a component of an adjacent cell surface, a transmitter from a synaptic partner, or via molecules released by other cells at varying distances. However, the discovery of exosomes changed the traditional theory. Evidence indicated that exosomes of different cellular origin acted on cells differently. For example, DCs stimulated proliferation of other $\mathrm{T}$ cells by engulfing $\mathrm{T}$ cell-derived exosomes [34]. Another report showed that DC-derived exosomes communicated with $\mathrm{T}$ cells by binding of LFA-1 and ICAM-1. Mittelbrunn et al. found that B cells transferred exosomes to $\mathrm{T}$ cells by immune synapse, which is a fairly surprising discovery [35]. In this study, we found that T cells did not engulf BMMC-exosomes, while a few exosomes adhered to $\mathrm{T}$ cells during the first 24 hours. Therefore, we speculated that BMMC-exosomes affected the differentiation of naive $\mathrm{CD} 4^{+}$ $\mathrm{T}$ cells by surface contact. However, the mechanism involving breakdown of BMMC-exosomes, release of their intraluminal components into the culture, and impact on $\mathrm{T}$ cells cannot be excluded. Based on previous studies, ligation of OX40L and OX40 on T cells facilitated naive $\mathrm{CD}^{+} \mathrm{T}$ cells to differentiate to Th2 cells [26]. In addition, mast cells and their exosomes expressed OX40L. Therefore, we selected anti-OX40L mAb to block OX40L on BMMC-exosomes to significantly decrease their ability to promote the differentiation of Th2 cells, at least partly. Such a result demonstrated that ligation of OX40L and OX40 was the molecular mechanism by which BMMC-exosomes enhanced the differentiation of Th2 cells. Possibly, other surface molecules or intraluminal components of BMMC-exosomes also participate in the regulatory process. Therefore, we speculated that BMMC-exosomes may act on distant $\mathrm{T}$ cells and other cells, circulating in the blood stream or lymph system [5]. In contrast to cells, the small-sized exosomes migrate from the secretary sites to the remote sites quickly and easily. Unlike soluble cytokines distributed in distant locations at a low level, exosomes disintegrate to release the contents locally at a relatively high level to sustain their function. Therefore, exosomes may communicate more efficiently than cells and soluble mediators.

In conclusion, we found that BMMC-exosomes promoted the proliferation of naive $\mathrm{CD} 4^{+} \mathrm{T}$ cells, although the ability was limited. However, the exosomes significantly facilitated the differentiation of naive $\mathrm{CD} 4^{+} \mathrm{T}$ cells to Th2 cells by ligation of OX40L and OX40 between BMMC-exosomes and $\mathrm{CD}^{+} \mathrm{T}$ cells and represents a novel mechanism of cell-to-cell communication. The study limitation relates to the experimental conditions in vitro and the result may not 


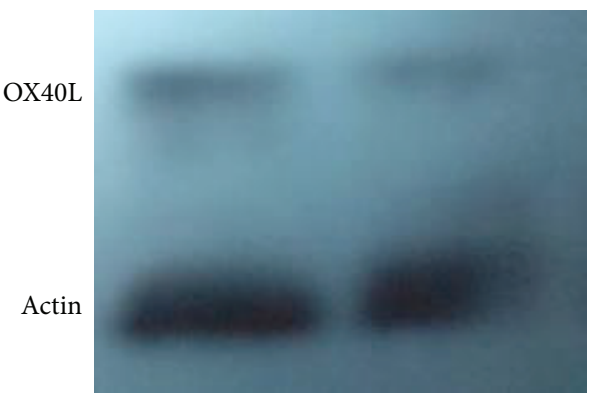

Cell exosome

(a)

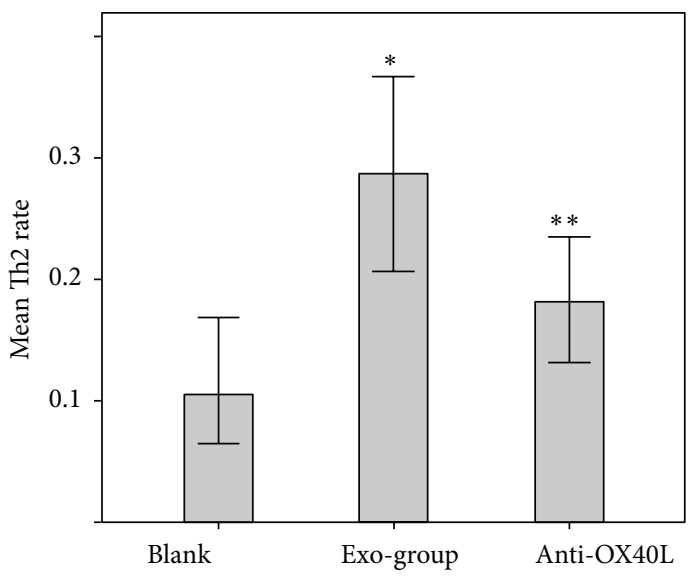

(c)

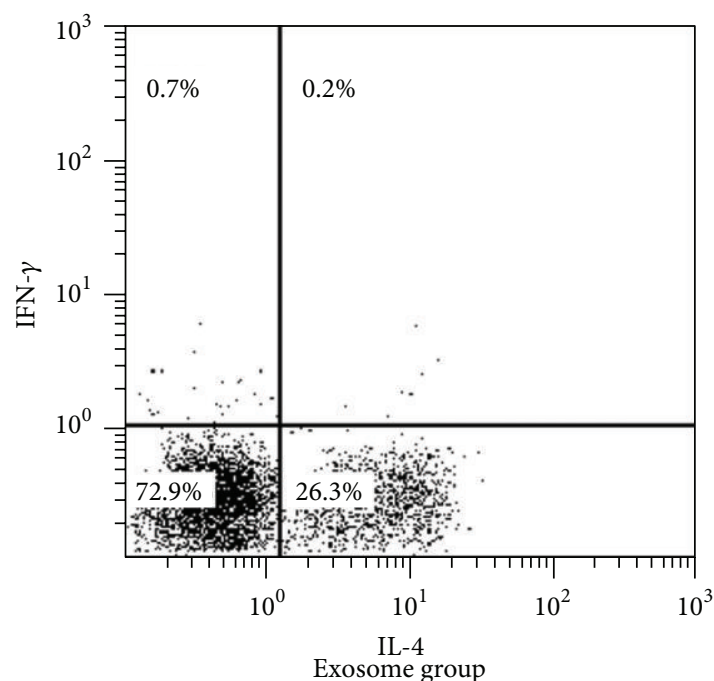

(e)

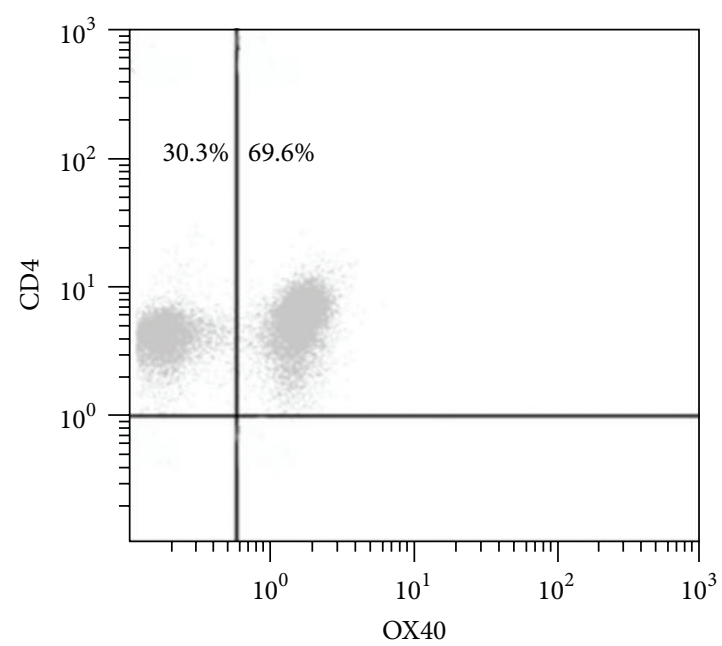

(b)

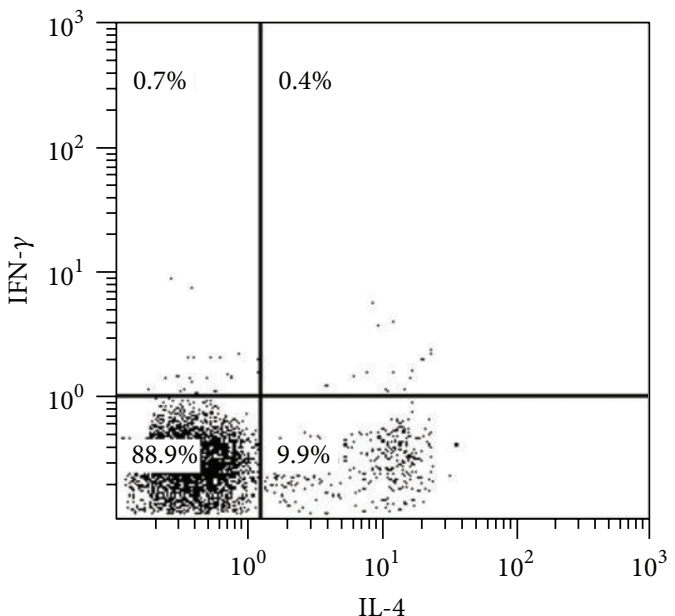

Blank control

(d)

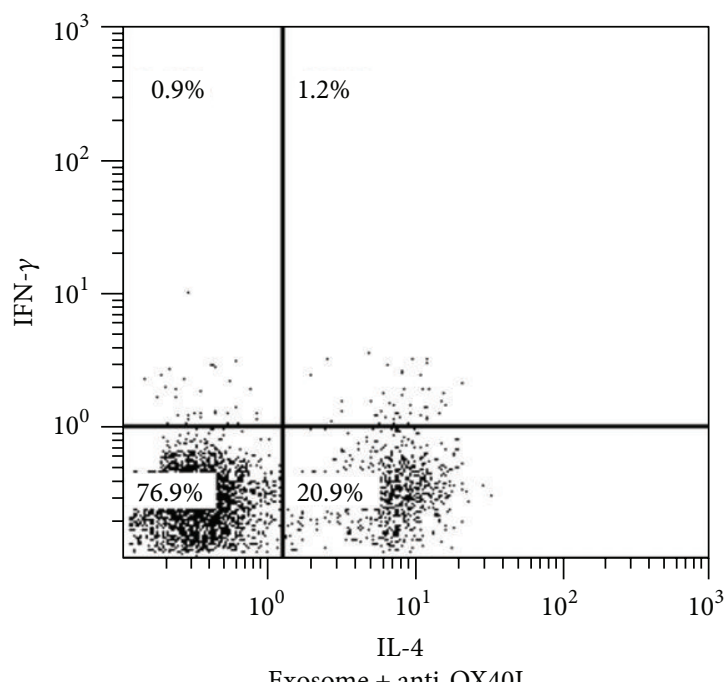

Exosome + anti-OX40L

(f)

FIGURE 5: Ligation of OX40L and OX40 promotes the differentiation of Th2 cells. (a) Western blot revealed the expression of OX40L in BMMCs and their exosomes, but a lower expression on exosomes; (b) flow cytometry showed that naive $\mathrm{CD} 4^{+} \mathrm{T}$ cells expressed OX40; (c) Th2 expression in all the T cells was expressed as mean \pm SD. $*$, compared with blank control, $p<0.05$. **, compared with exosome group, $p<0.05$. The results represented three independent experiments. (d-f) Addition of anti-OX40L Ab significantly but partially inhibited the differentiation of Th2 cells positive for IL- 4 . 
completely represent the conditions in vivo. Therefore, in vivo studies are needed to elucidate the effects of MC-exosomes on $\mathrm{CD} 4^{+} \mathrm{T}$ cells.

\section{Disclosure}

Fei $\mathrm{Li}$ is the first author and Yuping Wang is the co-first author.

\section{Conflict of Interests}

The authors declare no commercial or financial conflict of interests.

\section{Acknowledgments}

This work was supported by National Natural Science Foundation (no. 81072448, no. 81273276, and no. 81401335) and The Shanghai Municipal Science and Technology Commission Foundation (no. 11JC1410300 and no. 20124184).

\section{References}

[1] R. M. Johnstone, M. Adam, J. R. Hammond, L. Orr, and C. Turbide, "Vesicle formation during reticulocyte maturation. Association of plasma membrane activities with released vesicles (exosomes)," The Journal of Biological Chemistry, vol. 262, no. 19, pp. 9412-9420, 1987.

[2] V. Palanisamy, S. Sharma, A. Deshpande, H. Zhou, J. Gimzewski, and D. T. Wong, "Nanostructural and transcriptomic analyses of human saliva derived exosomes," PLoS ONE, vol. 5, no. 1, Article ID e8577, 2010.

[3] C. Admyre, S. M. Johansson, K. R. Qazi et al., "Exosomes with immune modulatory features are present in human breast milk," Journal of Immunology, vol. 179, no. 3, pp. 1969-1978, 2007.

[4] A. Cheruvanky, H. Zhou, T. Pisitkun et al., "Rapid isolation of urinary exosomal biomarkers using a nanomembrane ultrafiltration concentrator," The American Journal of PhysiologyRenal Physiology, vol. 292, no. 5, pp. F1657-F1661, 2007.

[5] C. Yang, M. A. Ruffner, S.-H. Kim, and P. D. Robbins, "Plasmaderived MHC class II+ exosomes from tumor-bearing mice suppress tumor antigen-specific immune responses," European Journal of Immunology, vol. 42, no. 7, pp. 1778-1784, 2012.

[6] J. Nilsson, J. Skog, A. Nordstrand et al., "Prostate cancer-derived urine exosomes: a novel approach to biomarkers for prostate cancer," British Journal of Cancer, vol. 100, no. 10, pp. 1603-1607, 2009.

[7] C. Subra, D. Grand, K. Laulagnier et al., "Exosomes account for vesicle-mediated transcellular transport of activatable phospholipases and prostaglandins," Journal of Lipid Research, vol. 51, no. 8, pp. 2105-2120, 2010.

[8] J. Conde-Vancells, E. Rodriguez-Suarez, N. Embade et al., "Characterization and comprehensive proteome profiling of exosomes secreted by hepatocytes," Journal of Proteome Research, vol. 7, no. 12, pp. 5157-5166, 2008.

[9] J. L. Welton, S. Khanna, P. J. Giles et al., "Proteomics analysis of bladder cancer exosomes," Molecular and Cellular Proteomics, vol. 9, no. 6, pp. 1324-1338, 2010.

[10] R. Wubbolts, R. S. Leckie, P. T. M. Veenhuizen et al., "Proteomic and biochemical analyses of human B cell-derived exosomes: potential implications for their function and multivesicular body formation," The Journal of Biological Chemistry, vol. 278, no. 13, pp. 10963-10972, 2003.

[11] C. Villarroya-Beltri, C. Gutiérrez-Vázquez, F. Sánchez-Madrid, and M. Mittelbrunn, "Analysis of microRNA and protein transfer by exosomes during an immune synapse," Methods in Molecular Biology, vol. 1024, pp. 41-51, 2013.

[12] S. M. Johansson, C. Admyre, A. Scheynius, and S. Gabrielsson, "Different types of in vitro generated human monocyte-derived dendritic cells release exosomes with distinct phenotypes," Immunology, vol. 123, no. 4, pp. 491-499, 2008.

[13] A. Carroll-Portillo, Z. Surviladze, A. Cambi, D. S. Lidke, and B. S. Wilson, "Mast cell synapses and exosomes: membrane contacts for information exchange," Frontiers in Immunology, vol. 3, article 46, Article ID Article 46, 2012.

[14] R. Mears, R. A. Craven, S. Hanrahan et al., "Proteomic analysis of melanoma-derived exosomes by two-dimensional polyacrylamide gel electrophoresis and mass spectrometry," Proteomics, vol. 4, no. 12, pp. 4019-4031, 2004.

[15] H. Valadi, K. Ekström, A. Bossios, M. Sjöstrand, J. J. Lee, and J. O. Lötvall, "Exosome-mediated transfer of mRNAs and microRNAs is a novel mechanism of genetic exchange between cells," Nature Cell Biology, vol. 9, no. 6, pp. 654-659, 2007.

[16] X. Yang, S. Meng, H. Jiang, C. Zhu, and W. Wu, "Exosomes derived from immature bone marrow dendritic cells induce tolerogenicity of intestinal transplantation in rats," Journal of Surgical Research, vol. 171, no. 2, pp. 826-832, 2011.

[17] N. Prado, E. G. Marazuela, E. Segura et al., "Exosomes from bronchoalveolar fluid of tolerized mice prevent allergic reaction," Journal of Immunology, vol. 181, no. 2, pp. 1519-1525, 2008.

[18] L. Zitvogel, A. Regnault, A. Lozier et al., "Eradication of established murine tumors using a novel cell-free vaccine: dendritic cell-derived exosomes," Nature Medicine, vol. 4, no. 5, pp. 594$600,1998$.

[19] D. Skokos, H. G. Botros, C. Demeure et al., "Mast cell-derived exosomes induce phenotypic and functional maturation of dendritic cells and elicit specific immune responses in vivo," Journal of Immunology, vol. 170, no. 6, pp. 3037-3045, 2003.

[20] C. Thery, S. Amigorena, G. Raposo, and A. Clayton, "Isolation and characterization of exosomes from cell culture supernatants and biological fluids," Current Protocols in Cell Biology, chapter 3: unit 3.22, 2006.

[21] A. Lorentz, I. Klopp, T. Gebhardt, M. P. Manns, and S. C. Bischoff, "Role of activator protein 1 , nuclear factor- $\kappa \mathrm{B}$, and nuclear factor of activated $\mathrm{T}$ cells in IgE receptor-mediated cytokine expression in mature human mast cells," Journal of Allergy and Clinical Immunology, vol. 111, no. 5, pp. 1062-1068, 2003.

[22] N. Depinay, F. Hacini, W. Beghdadi, R. Peronet, and S. Mécheri, "Mast cell-dependent down-regulation of antigenspecific immune responses by mosquito bites," The Journal of Immunology, vol. 176, no. 7, pp. 4141-4146, 2006.

[23] S. Nakae, H. Suto, M. Kakurai, J. D. Sedgwick, M. Tsai, and S. J. Galli, "Mast cells enhance T cell activation: importance of mast cell-derived TNF," Proceedings of the National Academy of Sciences of the United States of America, vol. 102, no. 18, pp. 64676472, 2005.

[24] A. E. Morelli, A. T. Larregina, W. J. Shufesky et al., "Endocytosis, intracellular sorting, and processing of exosomes by dendritic cells," Blood, vol. 104, no. 10, pp. 3257-3266, 2004. 
[25] E. Segura, C. Nicco, B. Lombard et al., "ICAM-1 on exosomes from mature dendritic cells is critical for efficient naive T-cell priming," Blood, vol. 106, no. 1, pp. 216-223, 2005.

[26] Y. Ohshima, L.-P. Yang, T. Uchiyama et al., "OX40 costimulation enhances interleukin-4 (IL-4) expression at priming and promotes the differentiation of naive human $\mathrm{CD} 4^{+} \mathrm{T}$ cells into high IL- 4-producing effectors," Blood, vol. 92, no. 9, pp. 3338$3345,1998$.

[27] J.-I. Kashiwakura, H. Yokoi, H. Saito, and Y. Okayama, "T cell proliferation by direct cross-talk between OX40 ligand on human mast cells and OX40 on human T cells: comparison of gene expression profiles between human tonsillar and lungcultured mast cells," The Journal of Immunology, vol. 173, no. 8, pp. 5247-5257, 2004.

[28] R. Malaviya, T. Ikeda, E. Ross, and S. N. Abraham, "Mast cell modulation of neutrophil influx and bacterial clearance at sites of infection through TNF- $\alpha$," Nature, vol. 381, no. 6577, pp. 7780, 1996.

[29] S. I. Da'as, A. J. Coombs, T. B. Balci, C. A. Grondin, A. A. Ferrando, and J. N. Berman, "The zebrafish reveals dependence of the mast cell lineage on Notch signaling in vivo," Blood, vol. 119, no. 15, pp. 3585-3594, 2012.

[30] H.-W. Wang, N. Tedla, A. R. Lloyd, D. Wakefield, and H. P. McNeil, "Mast cell activation and migration to lymph nodes during induction of an immune response in mice," Journal of Clinical Investigation, vol. 102, no. 8, pp. 1617-1626, 1998.

[31] V. Dimitriadou, S. Mécheri, M. Koutsilieris, W. Fraser, R. Al-Daccak, and W. Mourad, "Expression of functional major histocompatility complex class II molecules on HMC-1 human mast cells," Journal of Leukocyte Biology, vol. 64, no. 6, pp. 791799, 1998.

[32] Y.-S. Kim, H.-M. Ko, N.-I. Kang et al., "Mast cells play a key role in the development of late airway hyperresponsiveness through TNF- $\alpha$ in a murine model of asthma," European Journal of Immunology, vol. 37, no. 4, pp. 1107-1115, 2007.

[33] D. Skokos, S. Le Panse, I. Villa et al., "Mast cell-dependent B and $\mathrm{T}$ lymphocyte activation is mediated by the secretion of immunologically active exosomes," The Journal of Immunology, vol. 166, no. 2, pp. 868-876, 2001.

[34] P. Bastos-Amador, B. Pérez-Cabezas, N. Izquierdo-Useros et al., "Capture of cell-derived microvesicles (exosomes and apoptotic bodies) by human plasmacytoid dendritic cells," Journal of Leukocyte Biology, vol. 91, no. 5, pp. 751-758, 2012.

[35] M. Mittelbrunn, C. Gutiérrez-Vázquez, C. Villarroya-Beltri et al., "Unidirectional transfer of microRNA-loaded exosomes from T cells to antigen-presenting cells," Nature Communications, vol. 2, article 282, 2011. 


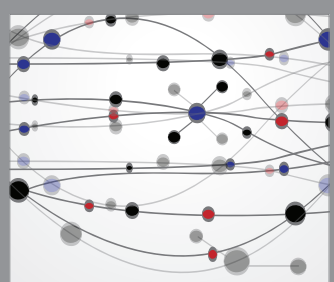

The Scientific World Journal
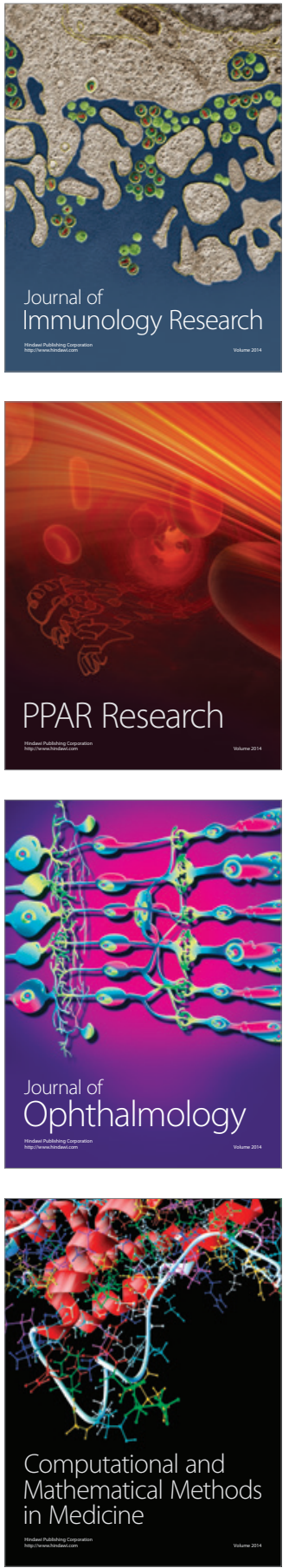

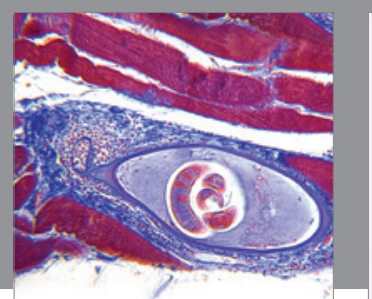

Gastroenterology Research and Practice

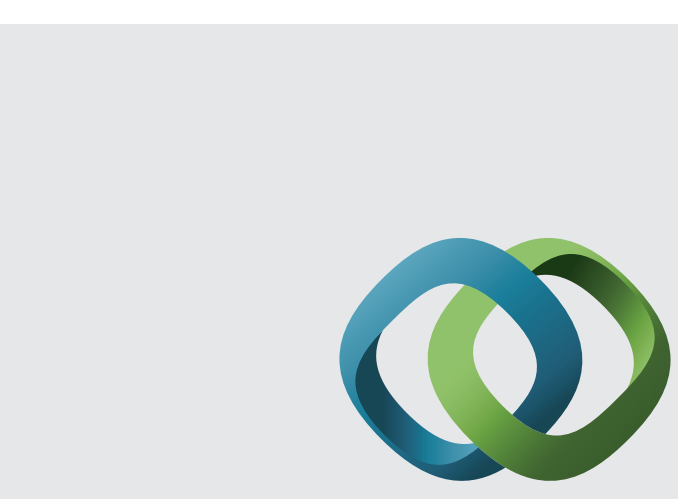

\section{Hindawi}

Submit your manuscripts at

http://www.hindawi.com
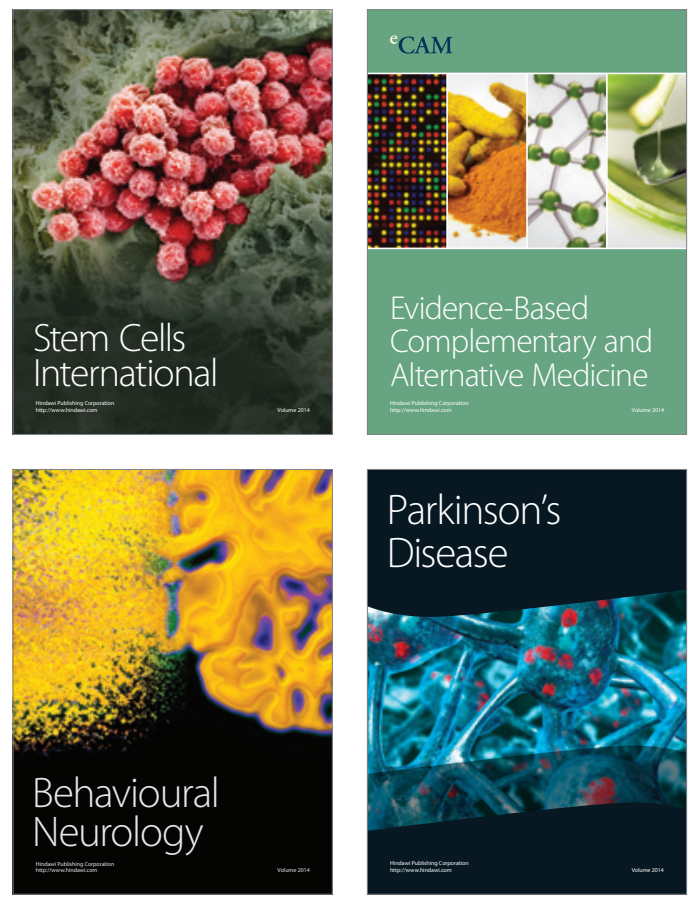
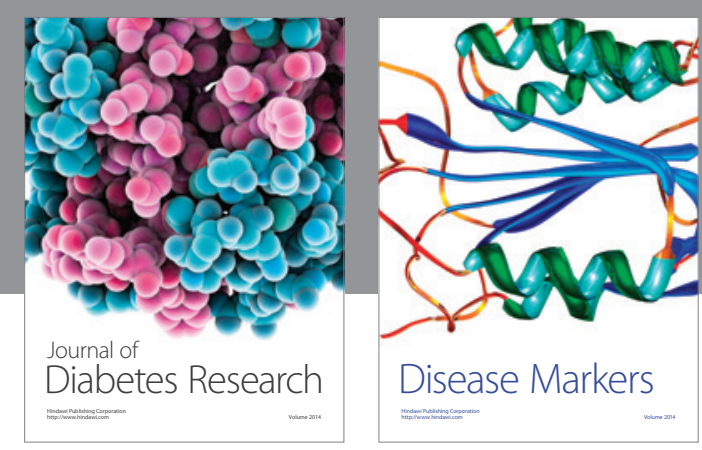

Disease Markers
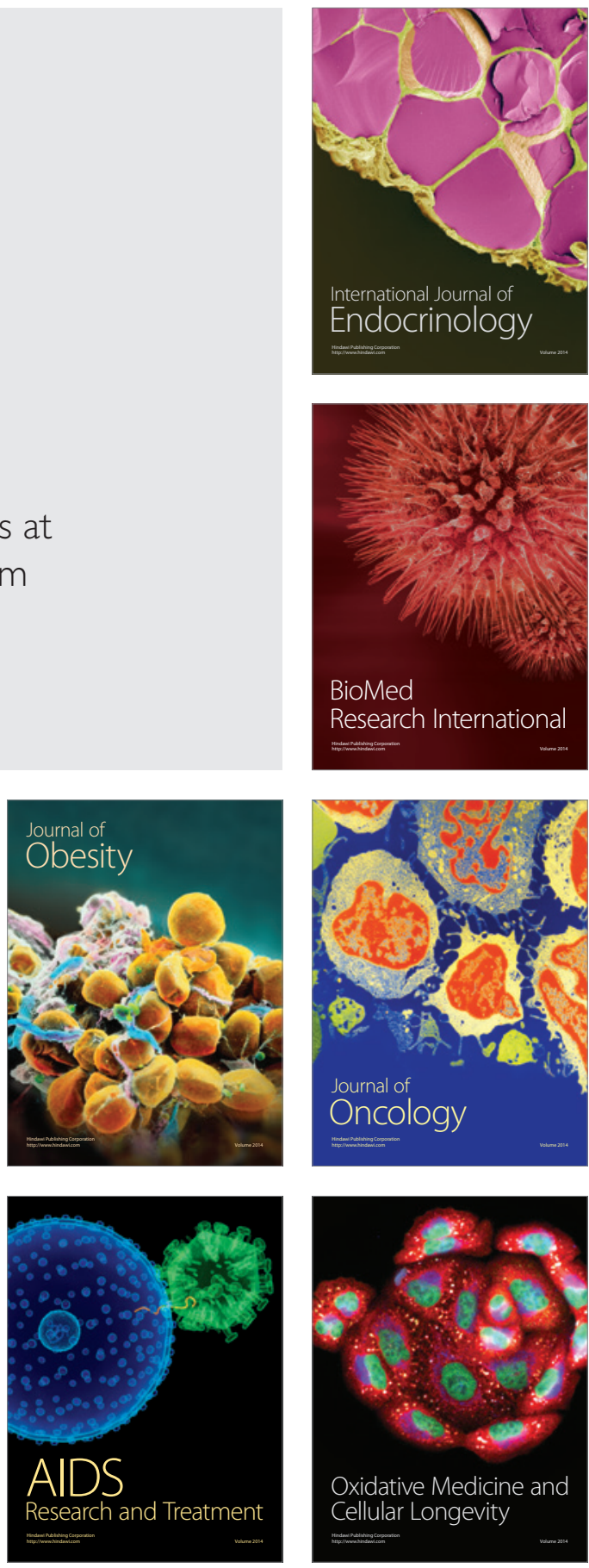